AFRL-IF-RS-TR-2003-46

Final Technical Report

March 2003

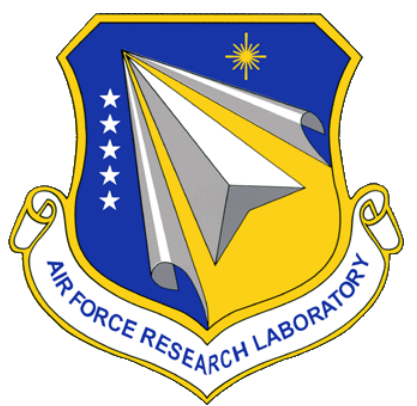

\title{
STORY-BASED INFORMATION PROCESSING FOR MILITARY FIELD APPLICATIONS: A FEASIBILITY STUDY
}

\author{
Duke University
}

Sponsored by

Defense Advanced Research Projects Agency

DARPA Order No. L140

The views and conclusions contained in this document are those of the authors and should not be interpreted as necessarily representing the official policies, either expressed or implied, of the Defense Advanced Research Projects Agency or the U.S. Government.

\author{
AIR FORCE RESEARCH LABORATORY \\ INFORMATION DIRECTORATE \\ ROME RESEARCH SITE \\ ROME, NEW YORK
}


This report has been reviewed by the Air Force Research Laboratory, Information Directorate, Public Affairs Office (IFOIPA) and is releasable to the National Technical Information Service (NTIS). At NTIS it will be releasable to the general public, including foreign nations.

AFRL-IF-RS-TR-2003-46 has been reviewed and is approved for publication.

APPROVED:

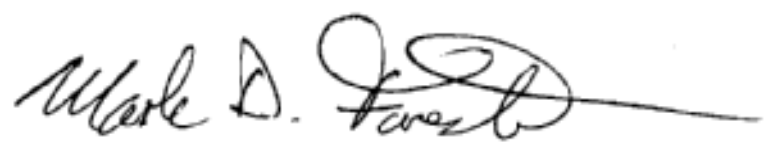

MARK D. FORESTI

Project Engineer

FOR THE DIRECTOR:

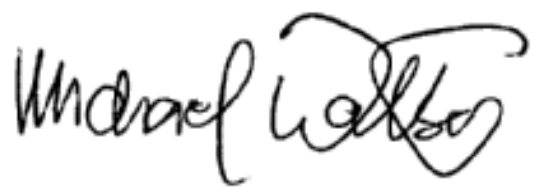

MICHAEL L. TALBERT, Maj., USAF

Technical Advisor, Information Technology Division Information Directorate 


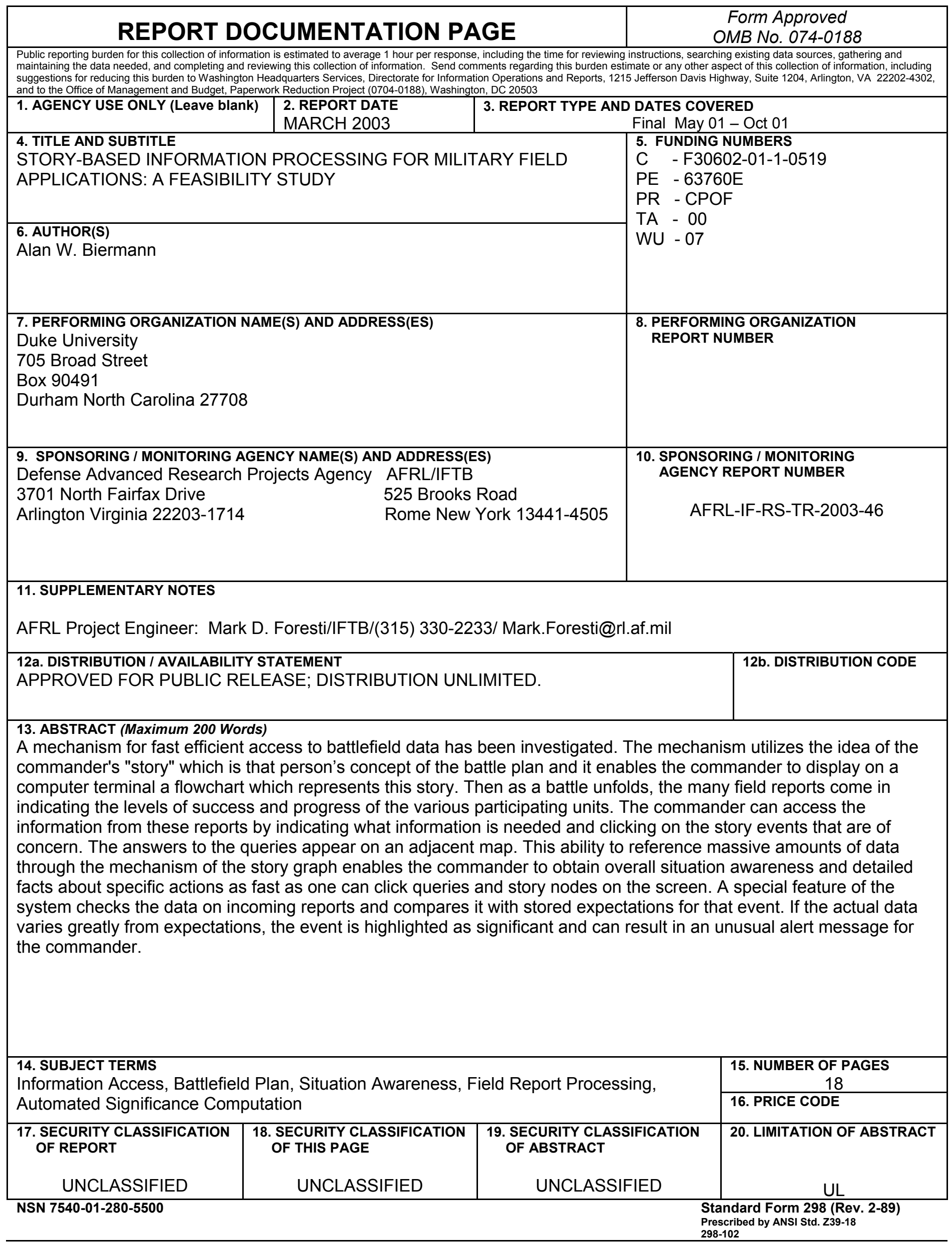




\section{Table of Contents:}

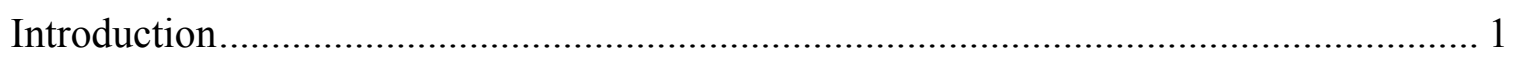

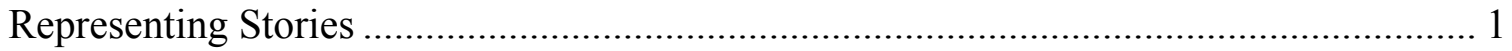

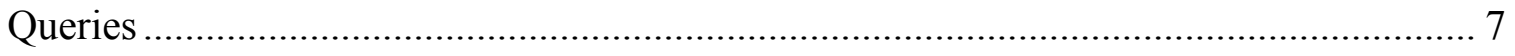

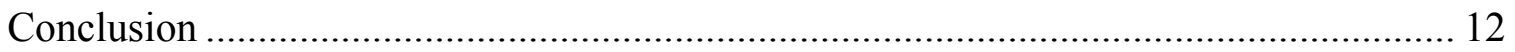

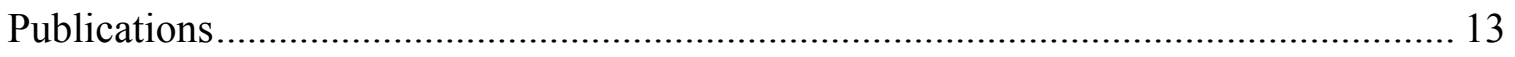

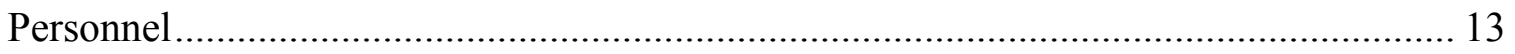

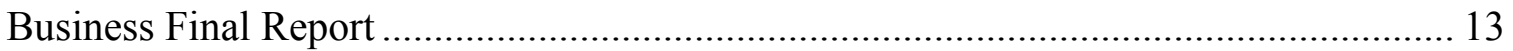




\section{List of Figures:}

Figure 1: Story Representation..... ....................................................................... 2

Figure 2: Story Representation with Map....... . ................................................... 4

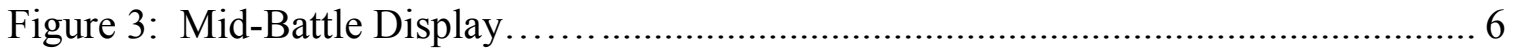

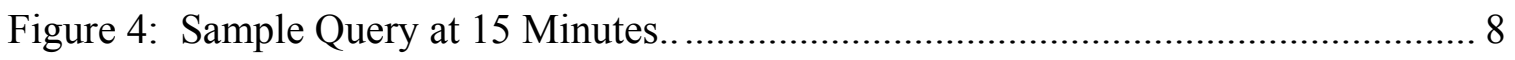

Figure 5: Sample Query at 105 Minutes............................................................... 9

Figure 6: Querying for Resistance Reports........................................................ 10

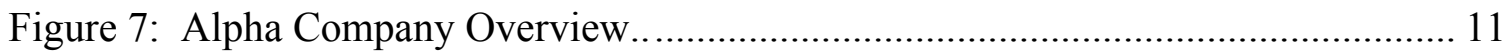




\section{Introduction}

In a meeting of the Command Post of the Future project in the Spring of 2000, General Keith Holcomb commented that commanders on the battlefield are becoming "dysfunctional" because of the flood of information. He described the level of confusion that can occur with the many officers on hand, the large number of incoming reports, the databases, and the maps with their "stickies" showing current actions. He said he envisions a military exercise as a "story" and that he wants information with respect to that story. If a particular unit is to go to a designated place and carry out a task, he wants to be able to select the dimensions of interest, level of success, contact with the enemy, casualties, etc. and make targeted queries about that action as it is progressing.

Our Program Director Ward Page asked my research group at Duke University to investigate these issues. How should one represent the "stories" that General Holcomb described and how should one enable the querying that he feels commanders need to be able to do to quickly assess all significant factors in an ongoing battle. Our project has addressed these issues.

\section{Representing Stories}

We decided that stories could be represented as node and transition graphs where nodes stand for significant events in a story and the transitions indicate movement from node to node. A node can represent any size unit from a single soldier to a battalion or larger. It can represent any event related to that unit from a long holding period to a momentary transition point along a sequence of events.

Figure 1 shows an example of a story involving four companies, Alpha A, Bravo $\mathrm{B}$, Charlie C, and Delta D. The graph represents an attack on a Vietnamese town (a central section of Hue) by these four companies, which began in the predawn hours and went forward for several hours. Transition labels show which unit is moving. A.2 indicates that the second platoon of alpha company is moving. The nodes show nominal information about each event and additional information can be found by clicking. The plan allows for midcourse decision-making. If conditions dictate, a unit might take one action or another. For example, in the upper right section of the figure, Charlie Company may start at the high school and remain there for 240 minutes or it may move to Back Building 3 by time 105 . 


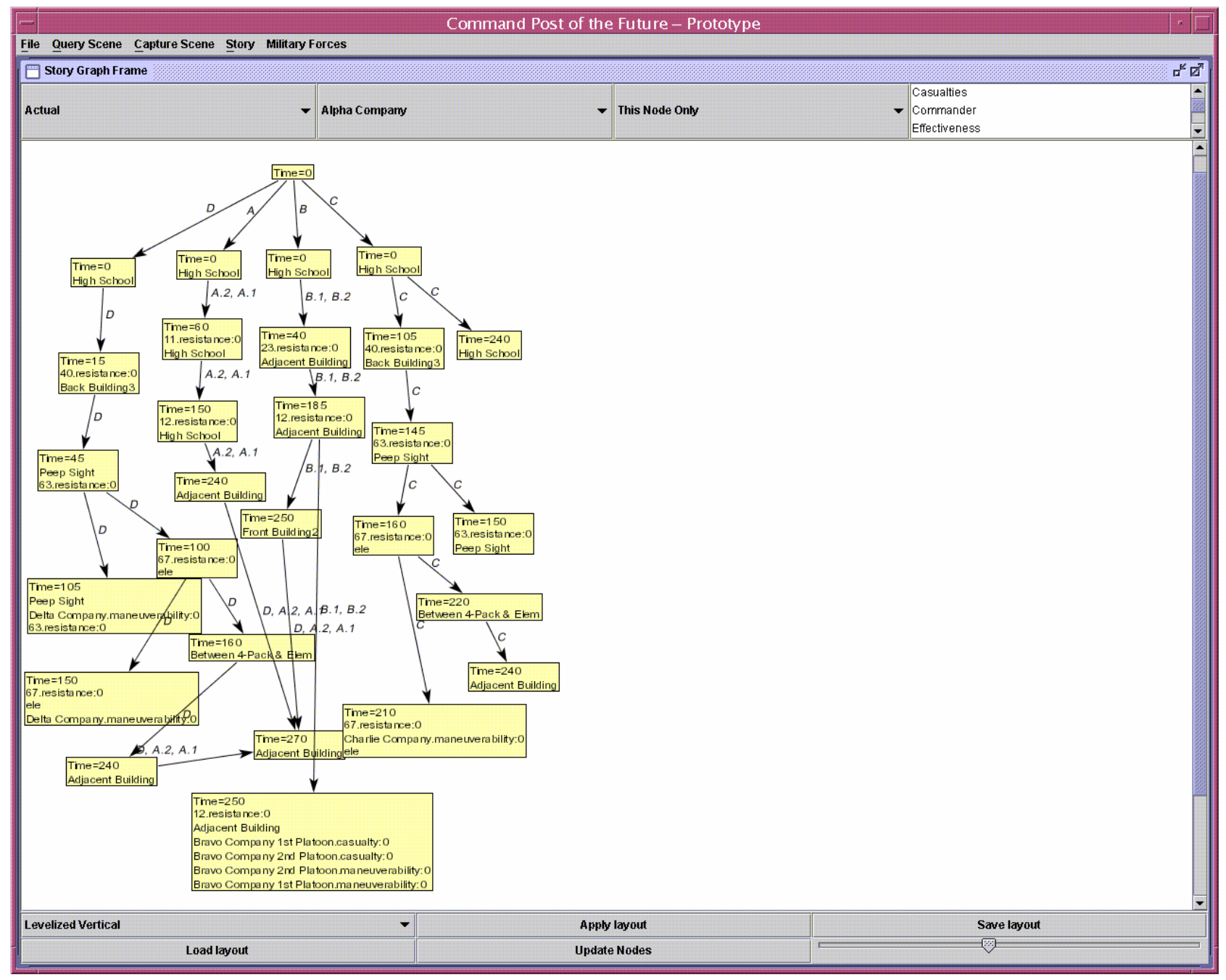

Figure 1: Story Representation. Above is a story representation of a simulation of the February 4, 1968 attack in Hue City. 
Our group used for our example military exercise, a simulation of the battle of Hue City on the morning of 4 February 1968. This simulation was carried out in the Spring of 2000 by the Command Post of the Future Project at the Institute for Defense Analysis in Alexandria, Virginia. In that simulation, General Keith Holcomb instructed a group of researchers in the Command Post of the Future project, who were acting as stand-in officers in the exercise, on how he proposed to do the attack. He gave specific instructions to each company commander on what he wanted them to do the next day in the attack and what options he wanted to consider along the way. This sequence of instructions was video recorded and later translated into our node and transition representation. Figure 1 shows the result. Each node includes the time in number of minutes after the launch of the action, usually the place of the action (such as the High School or the "Peep Sight" building), and possibly a special code indicating the expected level of enemy resistance. (Another CPOF-funded group at East Carolina University led by Professor Ronnie Smith has constructed a spoken language driven system that attempts to construct the representation of Figure 1 from the General's verbal instructions.)

After General Holcomb's orders were completed, the group carried out the simulation on a large model replica of Hue City, as it existed in February 1968. Occasionally, the participants were told that some event had occurred such as an incoming mortar that caused casualties. The officers of the affected units were required to modify their plan to account for such events. General Holcomb continuously queried the participants to determine their status and level of success and returned new commands to them to keep the total operation focused. All of these events were video recorded as well, and from them, my research group created a series of several hundredfield reports. These are the returned information from the field, which General Holcomb complained are flooding the commander with too much information.

Our system enables the commander to enter his or her personal concept of how the action is to take place and then to query the database of incoming reports to answer key questions with respect to this concept. Figure 2 shows the battle story plan at right and a map of the portion of Hue City of interest. The green nodes (blocks) at the top of the story graph show the currently active nodes at the beginning of the battle. Companies Alpha, Bravo, Charlie, and Delta are at the beginning point in the High School. In the upper left hand corner of the map, the large grey area is the High School and those companies are shown there. General Holcomb visited our laboratory in the summer of 2000 and advised us on our project. I asked whether the simulation of the Hue City battle that had been recorded was satisfactory for testing our system, and he indicated that he believed that it was. So this has provided that data that we have used in our study. It is relatively easy to load in data from another battle and show off our capabilities in that battle if necessary. 


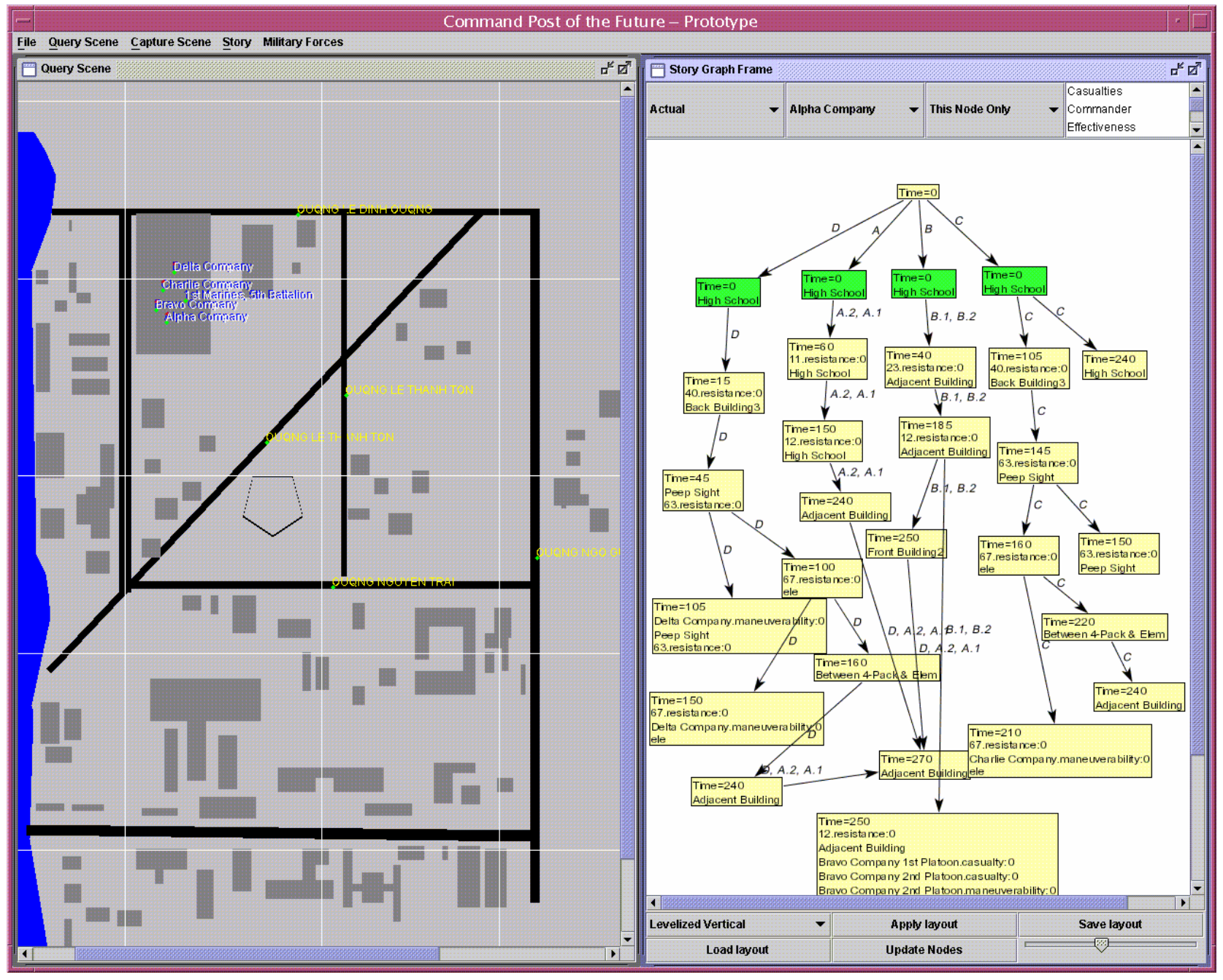

Figure 2: Story Representation with Map. The currently active nodes in green on the story graph show the beginning position for four companies, Alpha, Bravo, Charlie, and Delta. The large grey area on the upper left corner of the map shows the High School, which is occupied by four companies. 
Next, one can run the simulation on our system by starting the series of incoming field reports. These were the reports given by units during the simulated action. Figure 3 shows the appearance of the screen several simulated hours into the battle. Here Delta Company has advanced as far as the "Peep Sight" building. The green nodes on the story graph show the currently active nodes; the grey areas show the events that have occurred so far. The yellow nodes show steps of the plan that are yet to be executed. (The socalled "Peep Sight" Building was actually a hospital but was so named because its visual profile from above resembled a rifle peep sight. See the large grey area in Figure 2 just up and right from the lower left corner.) On the map, one can see the sources of some of the significant incoming reports indicated by red dots. Red dots with yellow surrounds indicate position where substantial enemy resistance has been reported. The numbers 2 and 3 in several locations indicate positions where casualties have occurred. The question marks are positions that have made requests of the Commander.

The window at the bottom of the map shows a listing of incoming field reports. On the left, all reports are shown in the scrollable window. On the right, only significant reports are given. These reports are automatically highlighted because they contain information that varies in major ways from the expectations for particular nodes. The story processing system includes an ability to select out unexpected information based on the commander's story and it can be queried directly. This information can also be used to return emergency messages for immediate attention of the commander. 


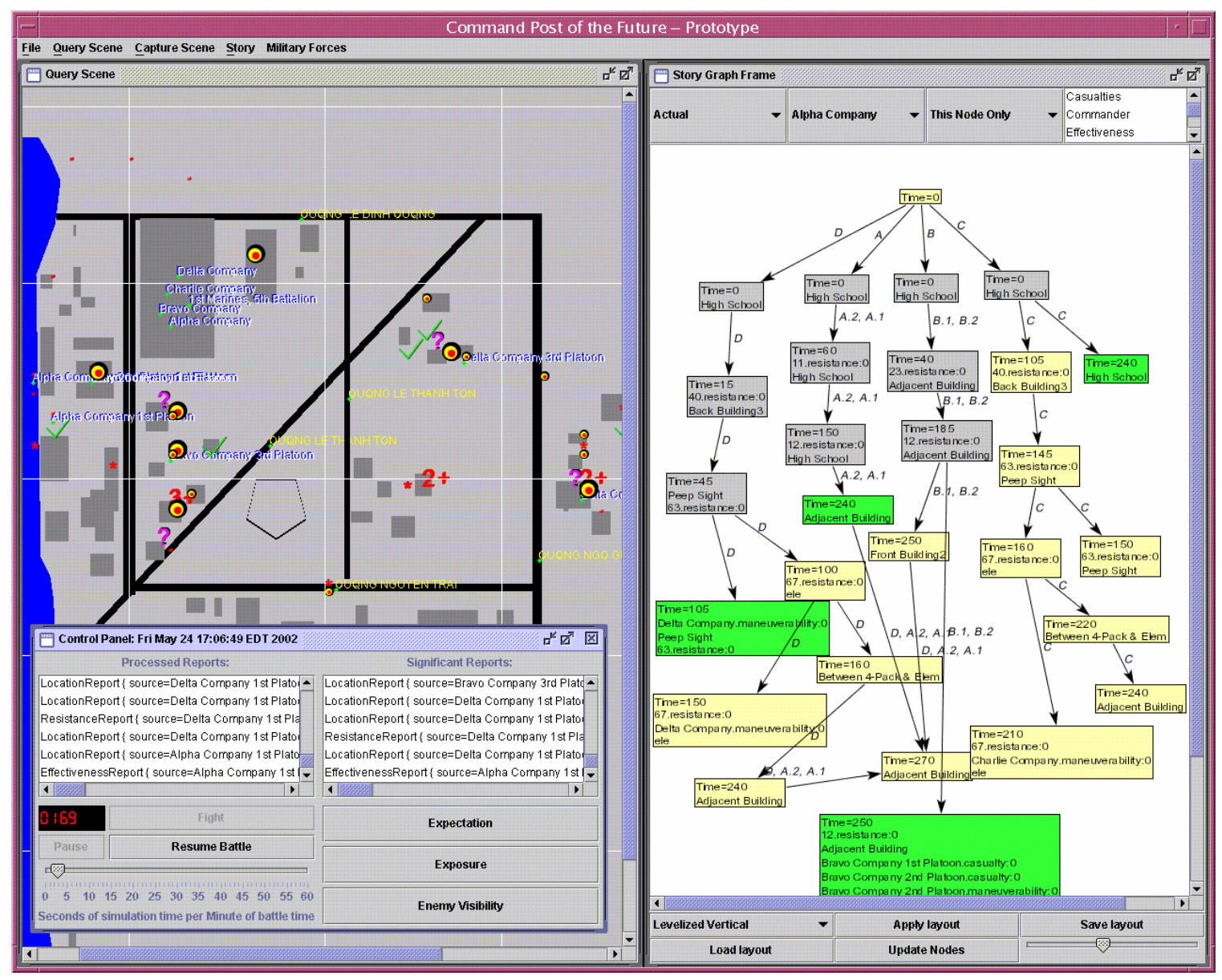

Figure 3: Mid-Battle Display. The system displays at an advanced position of the battle. Green nodes on the story show the current status of each company, grey nodes show completed actions, and yellow nodes show anticipated actions. The map shows significant unit locations, places of substantial enemy resistance (with yellow), and numbers of casualties with red numbers. The window at lower left has two listings; the leftmost one gives all incoming reports, the rightmost one gives only reports that have been marked as "significant" by the system. 


\section{Queries}

Next we examine the query handling ability of the system as requested by General Holcomb. In the upper right corner of the screen, one can see a field where the user can specify a dimension of interest. The field allows for the selection of any of these: Resistance, casualties, location, commander communication, force disposition, munitions, maneuverability, resource allocation, and effectiveness. (Most of our testing has involved the first three.) Then the system user can designate the node on the story and get an answer to the query for that particular unit at that particular time in the story. For example, the user may want to know what casualties Delta Company has incurred as the battle has unfolded. Then they would designate "casualties" in the upper right corner as shown and click whatever nodes on the Delta Company story path that seem to be important. We will assume here that the commander has been told that there have been casualties and the desire is to know exactly when and where these occurred. Figure 4 shows the result of a designating time node, $\mathrm{t}=15$, on the story graph. This checks casualties that may have occurred near the beginning of the action. It shows that there were two casualties in the area of the five pack buildings at that time. 


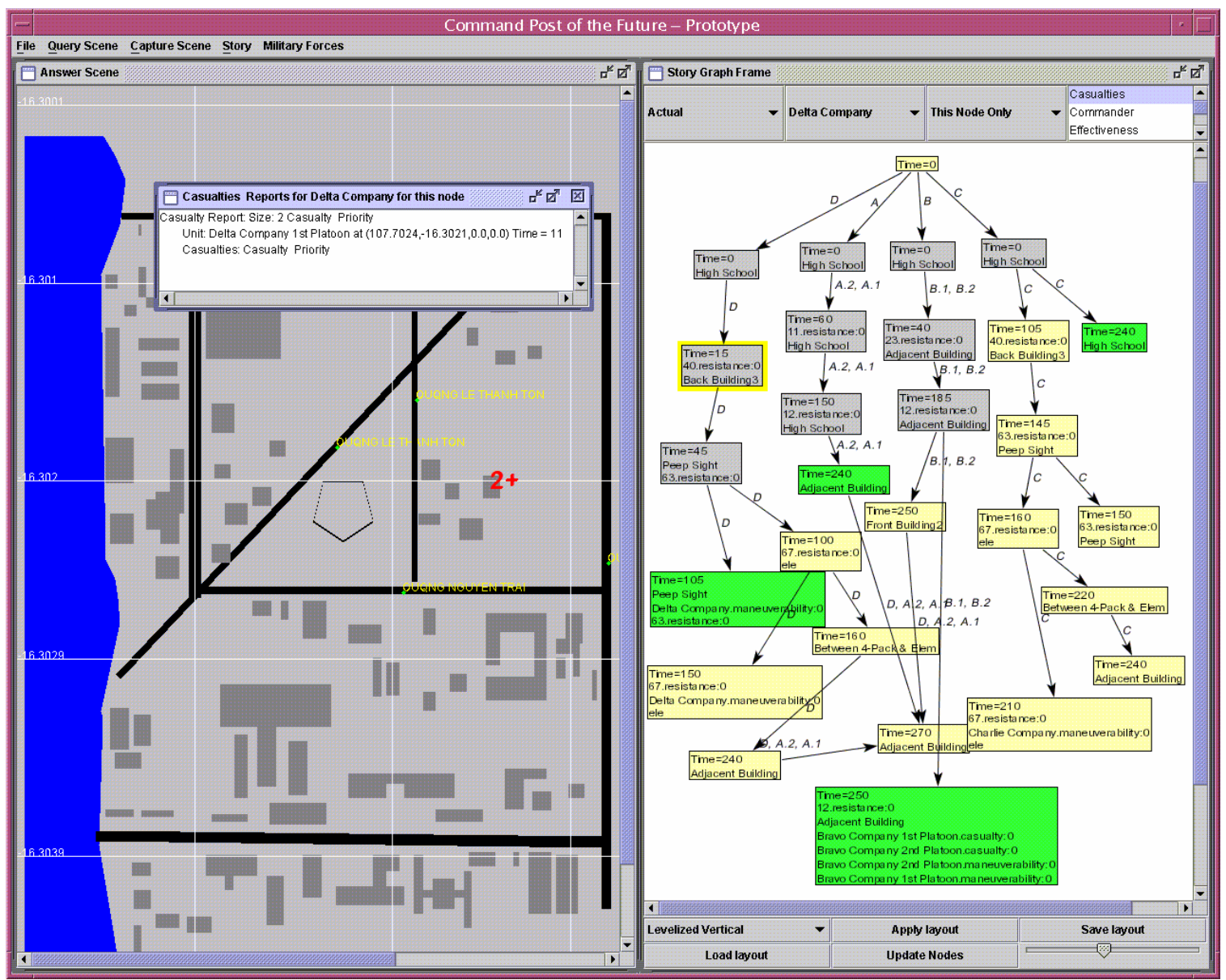

Figure 4: Sample Query at 15 Minutes. Querying for casualties for Delta Company at time 15 minutes.

Then one can check for casualties at the current part of the engagement, the green story node for Delta Company. Here the system indicates two additional casualties to the east of the five packs. 


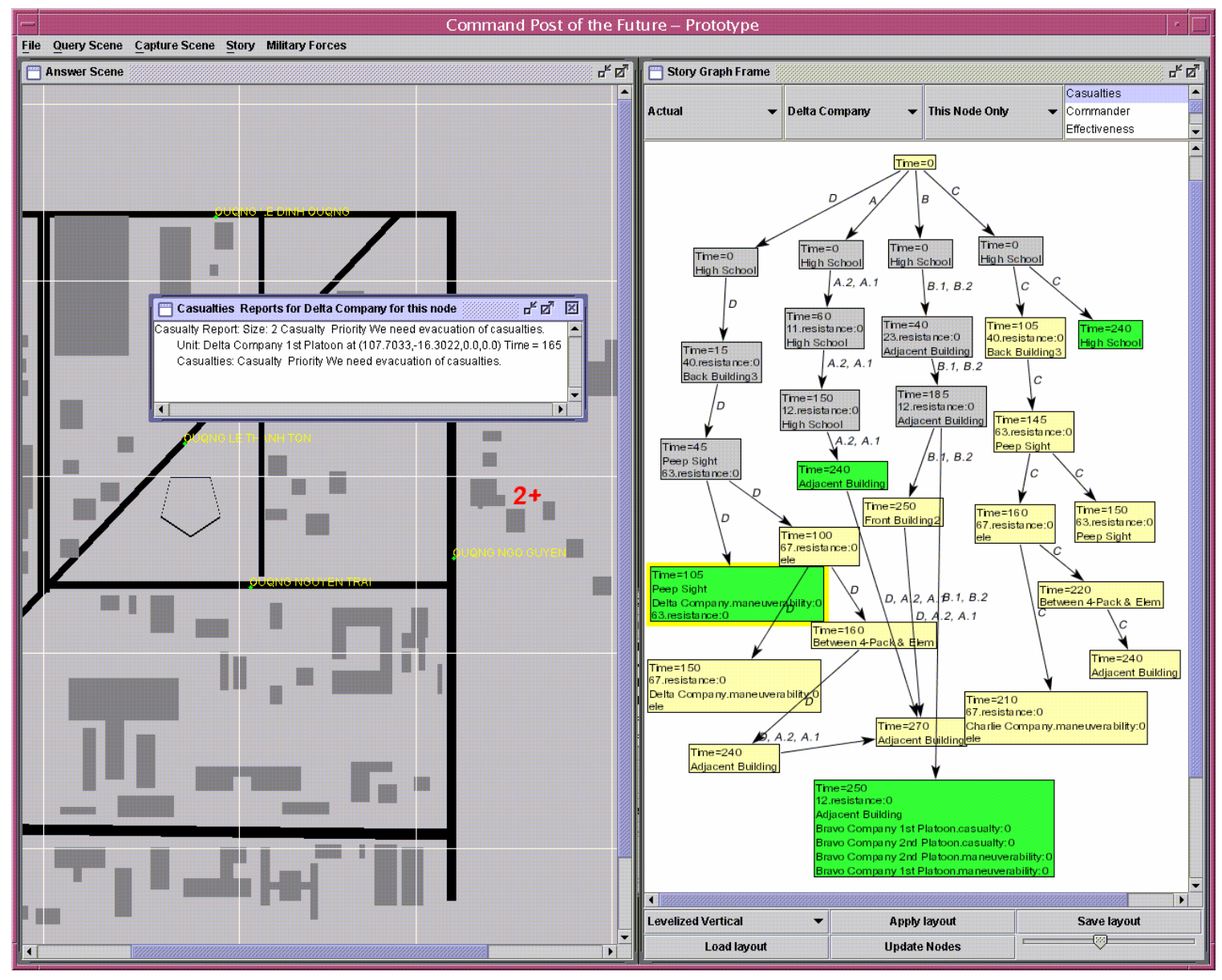

Figure 5: Sample Query at 105 Minutes. Querying the database for Delta Company at time 105 minutes. 
As an example of another query, Figure 6 shows an enemy resistance query for Delta Company. The response is a series of red dots with yellow surrounds where major resistance reports have been filed and the actual reports show up in the scrollable window given.

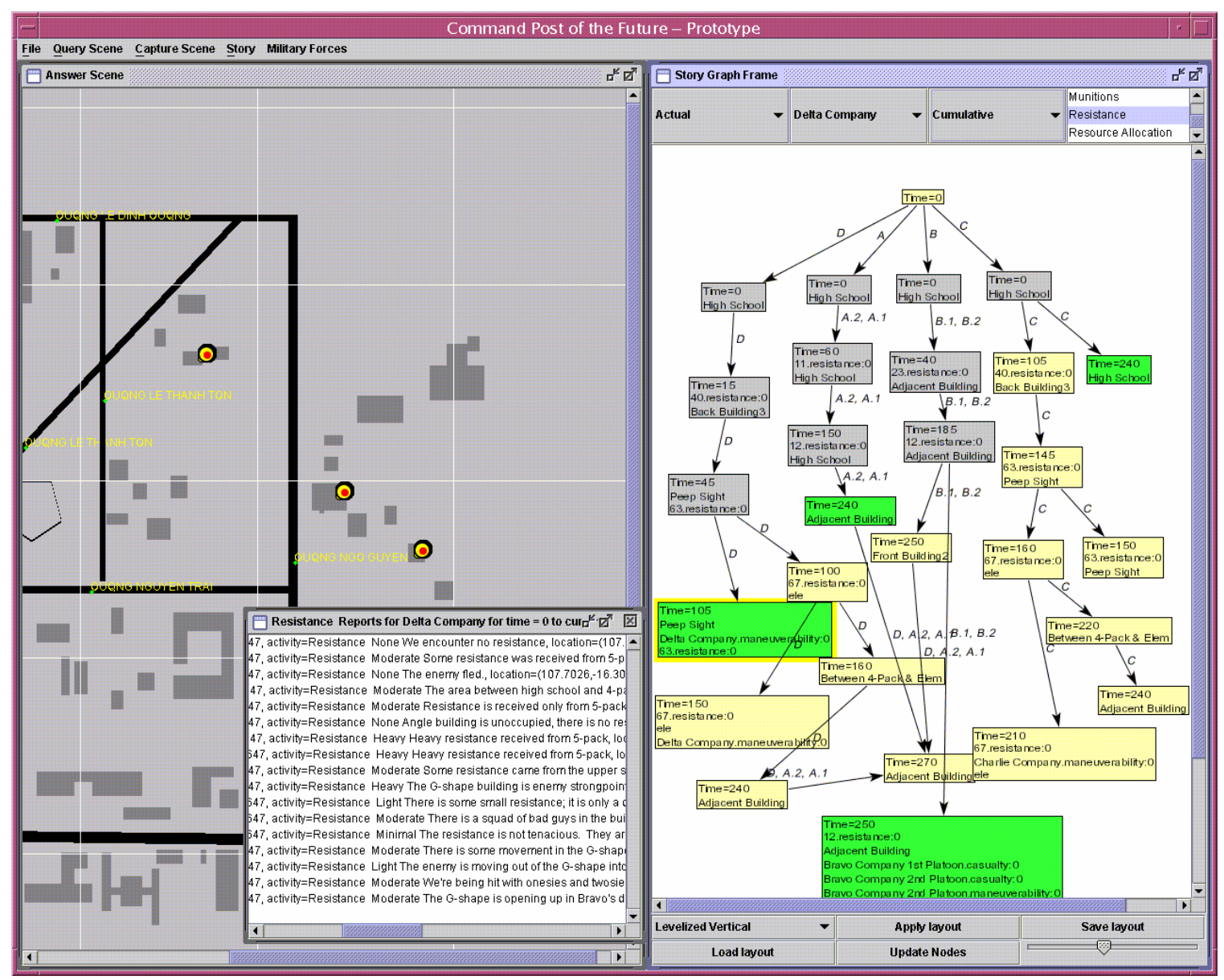

Figure 6: Querying for Resistance Reports. Querying the system for resistance reports for Delta Company. 
Finally, we might like to see a quick overview of a particular company's actions over a period of time. Figure 7 shows the set of all locations in the database for Alpha Company over a several hour period.

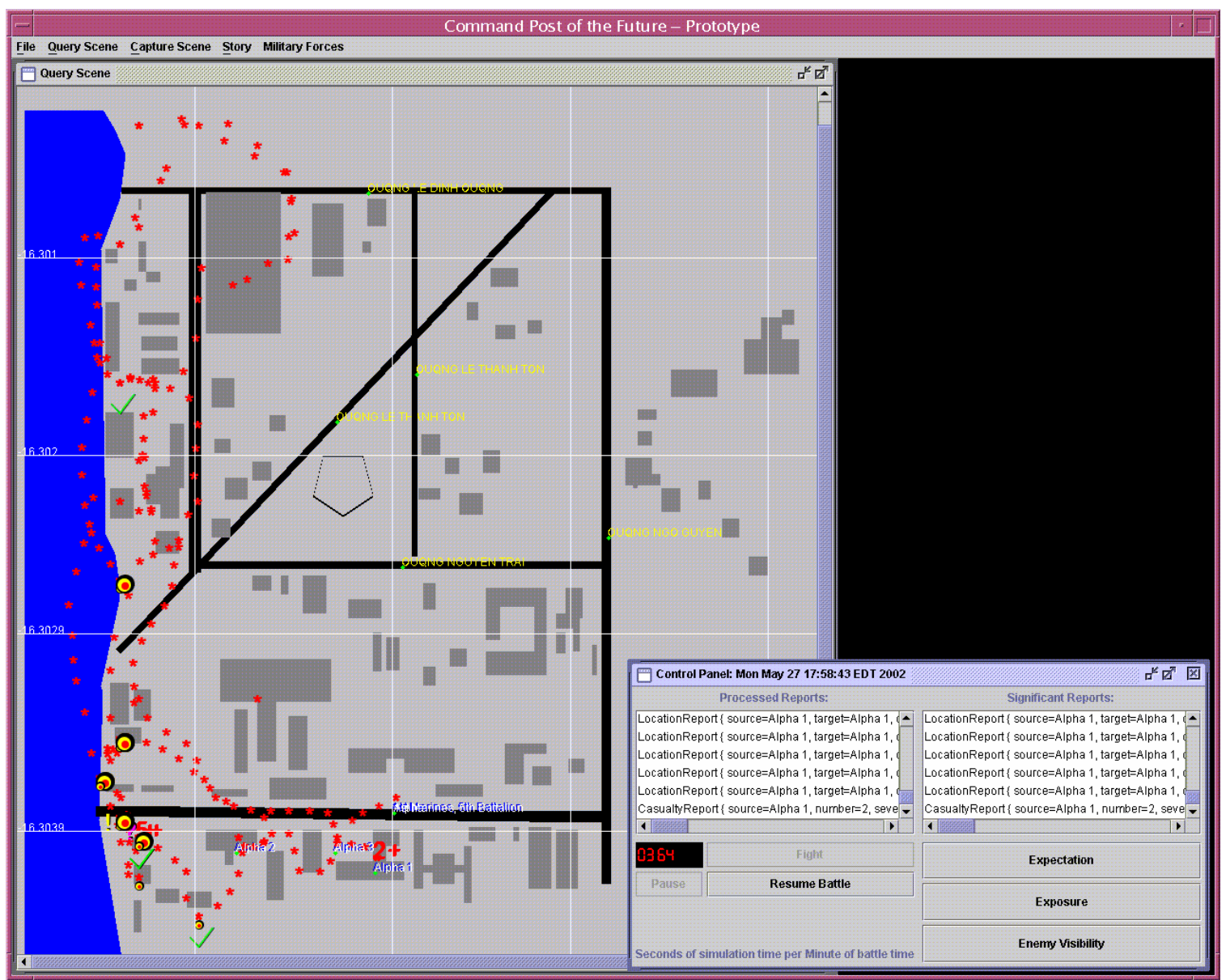

Figure 7: Alpha Company Overview. The set of all Alpha Company personnel locations in the database over a six-hour period. 


\section{Conclusion}

In conclusion, this tool is meant to meet the requirements given by General Keith Holcomb. Thousands of field reports may come in and the commanding officer will not necessarily want to see many of them. The officer will have a concept of what is going on and will want to query the database of reports with respect to that concept. Our tool enables immediate access to the information that is requested based on a few clicks on the screen. A representation of the answer appears on the map. However, the original text of the reports is also available. The system also has a significance-measuring feature that stores expectations of events and then highlights information that deviates from these expectations.

This project funded a feasibility study only and we are continuing the work. We would like to refine the capabilities shown here and then do user testing. We note that the query response is extremely fast so a commander will be able to gain broad situation awareness in a relatively short time. We would suggest that one can scan one dimension (query type) by hitting many story nodes in sequence. Checking the location, resistance, and casualty dimensions at many story nodes each should give an excellent overview of the situation. This might require about 30 second to 60 seconds for each of the three dimensions.

Our hope is to set up an experimental situation and then have some subjects examine the database through this tool. We will thus be in a position to evaluate and comment on its speed and effectiveness. We would also like to have additional advice from General Holcomb and others on the development of this tool and the opportunity to install whatever features may be needed. 


\section{Publications}

"Plan-based access to battlefield data in real time,"

Alan W. Biermann and students, in preparation.

\section{Personnel}

P. I. Alan W. Biermann

Students: Shannon Pollard, Justin Kalweit, Carl Miller, and Emma Buneci

\section{Business Final Report}

$\underline{\text { Date }}$

31 May 2001

30 June 2001

31 July 2001

31 August 2001

30 September 2001

31 October 2001
Amount obligated 50,000

Expended 0

$15,278.00$

$20,638.00$

$13,027.46$

0

1056.54 